\title{
El concepto de derivada y el modelo de Van Hiele en estudiantes de licenciatura en matemáticas e informática de la Universidad Francisco de Paula Santander.
}

No. 1

Enero-Diciembre 2015

\section{The concept of derivative and Van Hiele model students of bachelor in mathematics and informatics, University Francisco de Paula Santander.}

\author{
Edwin Giovanni Rodríguez-Pérez ${ }^{1}$
}

Forma de citar: E. G. Rodríguez. El concepto de derivada y el modelo de Van Hiele en estudiantes de licenciatura en matemáticas e informática de la Universidad Francisco de Paula Santander. Eco.Mat. 2015; 6(1): 43-49.

Recibido:

Junio 22 de 2014

Aceptado:

Agosto 25 de 2014

\section{Resumen}

Se realizó una caracterización de los niveles de razonamiento geométrico de Van Hiele aplicados al concepto de derivada en una muestra de 40 estudiantes de cálculo diferencial, integral y multivariado del plan de estudios de licenciatura en matemáticas e informática, en el cual se describe, determina y compara los diferentes niveles encontrados, la investigación realizada mostró que es posible aplicar los niveles de razonamiento geométrico del modelo educativo de los esposos Van Hiele aun concepto propio de la Matemática.

Palabras clave: Concepto de derivada, niveles de razonamiento geométrico, modelo, Van Hiele, Visualización, Reconocimiento, Análisis, Ordenación, Deducción Formal, Rigor.

\section{Summary}

There was realized a charectization of the levels geometric reasoning of Van Hiele,applied to the concept of derivate in a sample of 40 students of defferential, integral and multivaried calculation of study master's plan mathemathics and computer sciencie of the University Francisco de Paula Santander, in which it is discribed, determines and compares the different opposing levels, the realized investigation showed that it's possible to apply

Keywords: Concept of derivate, levels geometric reasoning, Display, model, Van Hiele, Recognition, Analysis, Management, Formal Deduction, Rigor.
${ }^{1}$ Licenciado en matemáticas e informática, Maestrante en gerencia educativa. gio.rodriguez.169@facebook.com

\section{Introducción}

desde sus inicios la enseñanza y aprendizaje de la matemática ha presentado constantes inconvenientes, en especial al trabajar temas específicos; según investigadores esto se evidencia al cometer equivocaciones que muestran las dificultades en: el pensamiento, el proceso de aprendizaje matemático y el lenguaje de representación (externa e interna), etc. En la universidad Francisco de Paula Santander los estudiantes de licenciatura en Matemáticas e Informática también presentaron problemas en la comprensión de conceptos matemáticos particularmente en la derivada, debido a diferentes 
Eco.Mat

Cúcuta-Colombia

Vol. 6

No. 1

Enero-Diciembre 2015

ISSN 1794-8231

ISSN 2462 - $8794 \mathrm{Web}$

PP: $43-49$

El proyecto aplico el modelo de Van Hiele al concepto de derivada para caracterizar los niveles de razonamiento geométrico en el que se encontraban los estudiantes de cálculo diferencial, integral y multivariado del plan de estudios de licenciatura en Matemática e Informática; en el que se plantearon algunas hipótesis de orientación a la investigación pero no fueron probadas o refutadas, midió las variables, los cinco niveles de razonamiento geométrico de este modelo: Visualización o Reconocimiento, Análisis, Ordenación o Clasificación, Deducción Formal y Rigor.

La investigación fue cuantitativa, exploratoria, descriptiva y de campo, utilizó como técnica de investigación un cuestionario Adhoc, que se aplicó a estudiantes del plan de estudios de licenciatura Matemáticas e Informática de la Universidad Francisco De Paula Santander, y tuvo a cabo las siguientes fases: Primera, diseño del instrumento y realización de la prueba piloto según los niveles de razonamiento geométrico, para la confiabilidad y validez del instrumento; Segunda, Aplicó la corrección del instrumento a la muestra de estudiantes de cálculo diferencial, integral y multivariado; Tercera: Proceso y análisis de la información recolectada mediante el uso de Microsoft Excel; Cuarta, Elaboración y corrección del informe final. En ella se cuantificaron los datos, se aplicó un muestreo de juicio y

\section{Materiales y Métodos}

\section{Modelo Educativo De Van Hiele}

El modelo educativo de Van Hiele se debe al matrimonio de Dina y Pierre Van Hiele y se formuló inicialmente para la enseñanza y el aprendizaje de la geometría elemental. Trabaja la idea básica "que el aprendizaje de la matemática y la geometría se hace pasando

por unos determinados niveles de pensamiento y conocimiento", "que no van asociados a la edad", y "que solo alcanzando un nivel se puede pasar al siguiente". El modelo está compuesto por dos partes:

I. Los niveles de razonamiento geométrico de Van Hiele: Que describen la forma como los estudiantes asimilan y realizan diversas actividades de razonamiento a partir de un concepto geométrico. El modelo determina cinco niveles que se clasifican en:

- Nivel I: de Visualización: Los objetos se perciben en su totalidad como una unidad, sin diferenciar sus atributos y componentes. Se describen figuras por su apariencia física mediante descripciones meramente visuales y se asemejan a elementos familiares del entorno; No se reconocen de forma explícita componentes y propiedades de los objetos.

- Nivel II: de Análisis: Se perciben las componentes y propiedades de los objetos y figuras. Esto se obtiene tanto desde la observación como de la experimentación. De una manera informal se pueden describir las figuras por sus propiedades; Experimentando con figuras $u$ objetos se pueden establecer nuevas propiedades.

- Nivel III: de Ordenación y clasificación: Se comprende, dan, ordenan y modifican las definiciones. Se realizan las clasificaciones lógicas de manera formal, el razonamiento matemático esta iniciado.

- Nivel IV: de Deducción formal: Se realizan deducciones y demostraciones lógicas y formales viendo su necesidad para justificar las proposiciones planteadas. Se comprenden y manejan las relaciones entre propiedades y se 
formalizan en sistemas axiomáticos. Se completa la formación del razonamiento matemático lógicoformal de los individuos.

- Nivel V: de Rigor: En él se conoce la existencia de diferentes sistemas axiomáticos y se trabajan la matemática en su forma abstracta sin necesidad de ejemplos concretos.

Estos niveles de razonamiento no están asociados a habilidades computacionales o al desarrollo biológico del individuo, más bien están en función de las experiencias de aprendizaje a las que ha sido expuesto un individuo a lo largo de su vida con respecto al concepto objeto de estudio.

II. Las fases de aprendizaje: Se postulan en el modelo cinco fases y, a continuación, se describen: están orientadas a ayudar a progresar a un estudiante desde un nivel de razonamiento inmediatamente superior, básicamente las fases constituyen un esquema para organizar la enseñanza, y se clasifican en:

- Fase 1. Información. Se busca que los estudiantes expliciten la información que tienen en su estructura cognitiva acerca del concepto objeto de estudio.

- Fase 2. Orientación dirigida. Se proponen actividades en las que el concepto se relacione con situaciones de la vida diaria

- Fase 3. Explicitación. Se aplican los conceptos para resolver problemas que correspondan a situaciones reales en diferentes contextos.

- Fase 4. Orientación libre. Se completa la red de relaciones que se comenzó a formar en las fases anteriores $y$ se adquiere el lenguaje propio del siguiente nivel de razonamiento
- Fase 5. Integración. El concepto estudiado se reorganiza y adquiere un nuevo significado. Se hace explicita la nueva red conceptual y el conjunto de habilidades de razonamiento adquiridos.

Generalizando, se dice que la capacidad de razonamiento geométrico de los individuos puede evolucionar a lo largo del tiempo pasando por diferentes grados de calidad.

\section{Resultados y Discusión}

La investigación aplicó un instrumento de prueba piloto; se realizó en un grupo de 5 (cinco) estudiantes de noveno semestre de licenciatura en Matemáticas e Informática; para mayor confiabilidad se ejecutó la validación por expertos con la colaboración de los docentes: Olga Lucy Rincón, Jairo Camacho Villamarin y Gloria Stella Alba Duran.

En los Resultados de la prueba piloto se tuvo en cuenta el tiempo empleado en el desarrollo de la misma, verificando fuera apta para resolverse en un periodo máximo de dos horas, en la solución los estudiantes identificaron el tema señalado en el cuestionario, reconociendo el tema fue trabajado en los primeros semestres de la carrera y se les dificultó reconocer la representación geométrica de la derivada, se pudo observar que los estudiantes se ubican en los cuatro primeros niveles de razonamiento geométrico del modelo de Van Hiele y que en el nivel de rigor los estudiantes presentaron dificultades en la actividad demostrativa, obteniendo solo un $5 \%$ de acierto en las preguntas de este nivel.

Seguido a esto, se estructuró el instrumento de prueba definitiva, se aplicó con la colaboración de los docentes Rosa Virginia 
Eco.Mat

Cúcuta-Colombia

Vol. 6

No. 1

Enero-Diciembre 201 ISSN 1794-8231

ISSN $2462-8794 \mathrm{Web}$ PP: 43-49
El concepto de derivada y el modelo de Van Hiele en estudiantes de licenciatura en matemáticas e informática de la Universidad Francisco de Paula Santander

Hernández, Nancy Velásquez y Jairo Camacho Villamarin; a 21 estudiantes de cálculo diferencial, 14 de cálculo integral y solo 5 estudiantes de cálculo multivariado, siendo un total de cuarenta estudiantes en consecuencia a la deserción escolar en estas asignaturas.

\section{Técnicas De Análisis E Interpretación}

En esta investigación la información recolectada se procesó en la hoja de cálculo y se utilizaron elementos de la estadística descriptiva, se realizaron gráficos de representación para facilitar el análisis de datos. Con los análisis obtenidos se realiza un documento en el procesador de textos con cuadros comparativos, descripción y puntuación de variables en los niveles de Van Hiele aplicados en el concepto de derivada.

Para realizar el cumplimiento de los objetivos a través del análisis de la información recolectada se realizó análisis individual de las preguntas por niveles de razonamiento geométrico de Van Hiele, y se comparan los resultados obtenidos por los estudiantes en las diferentes asignaturas.

Del mismo modo se realizó la caracterización de los niveles de razonamiento geométrico de Van Hiele en el concepto de derivada estableciendo el Porcentaje de respuestas correctas en los cinco niveles, obteniendo los siguientes resultados:

Tabla1. PORCENTAJE DE RESPUESTAS CORRECTAS EN LOS CINCO NIVELES

\begin{tabular}{|l|c|c|c|}
\hline \multirow{2}{*}{$\begin{array}{c}\text { NIVELES DE } \\
\text { RAZONAMIENTO } \\
\text { GEOMÉTRICO }\end{array}$} & \multicolumn{3}{|c|}{ PORCENTAJE DE ESTUDIANTES } \\
\cline { 2 - 4 } & $\begin{array}{c}\text { CALCULO } \\
\text { DIFERENCIAL }\end{array}$ & $\begin{array}{c}\text { CALCULO } \\
\text { INTEGRAL }\end{array}$ & $\begin{array}{c}\text { CALCULO } \\
\text { MULTIVARIADO }\end{array}$ \\
\hline NIVEL I & $54,3 \%$ & $62,9 \%$ & $72 \%$ \\
\hline NIVEL II & $53,3 \%$ & $54,3 \%$ & $68 \%$ \\
\hline NIVEL III & $31,0 \%$ & $53,6 \%$ & $65 \%$ \\
\hline NIVEL IV & $19,1 \%$ & $32,1 \%$ & $30 \%$ \\
\hline
\end{tabular}

Fuente: Rodríguez Pérez

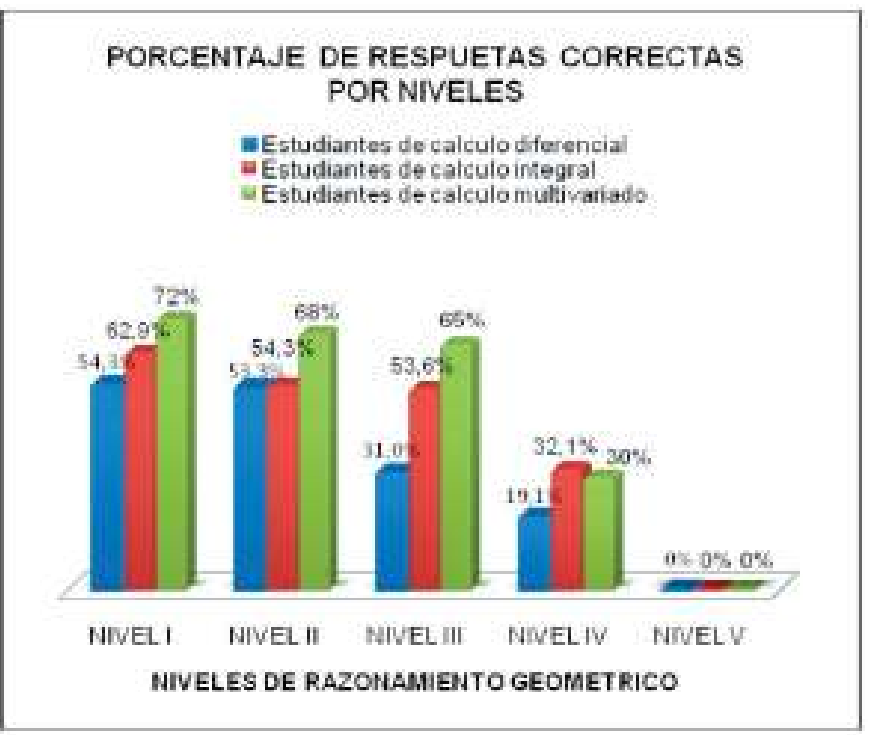

Figura1. Porcentaje de respuestas correctas por niveles 


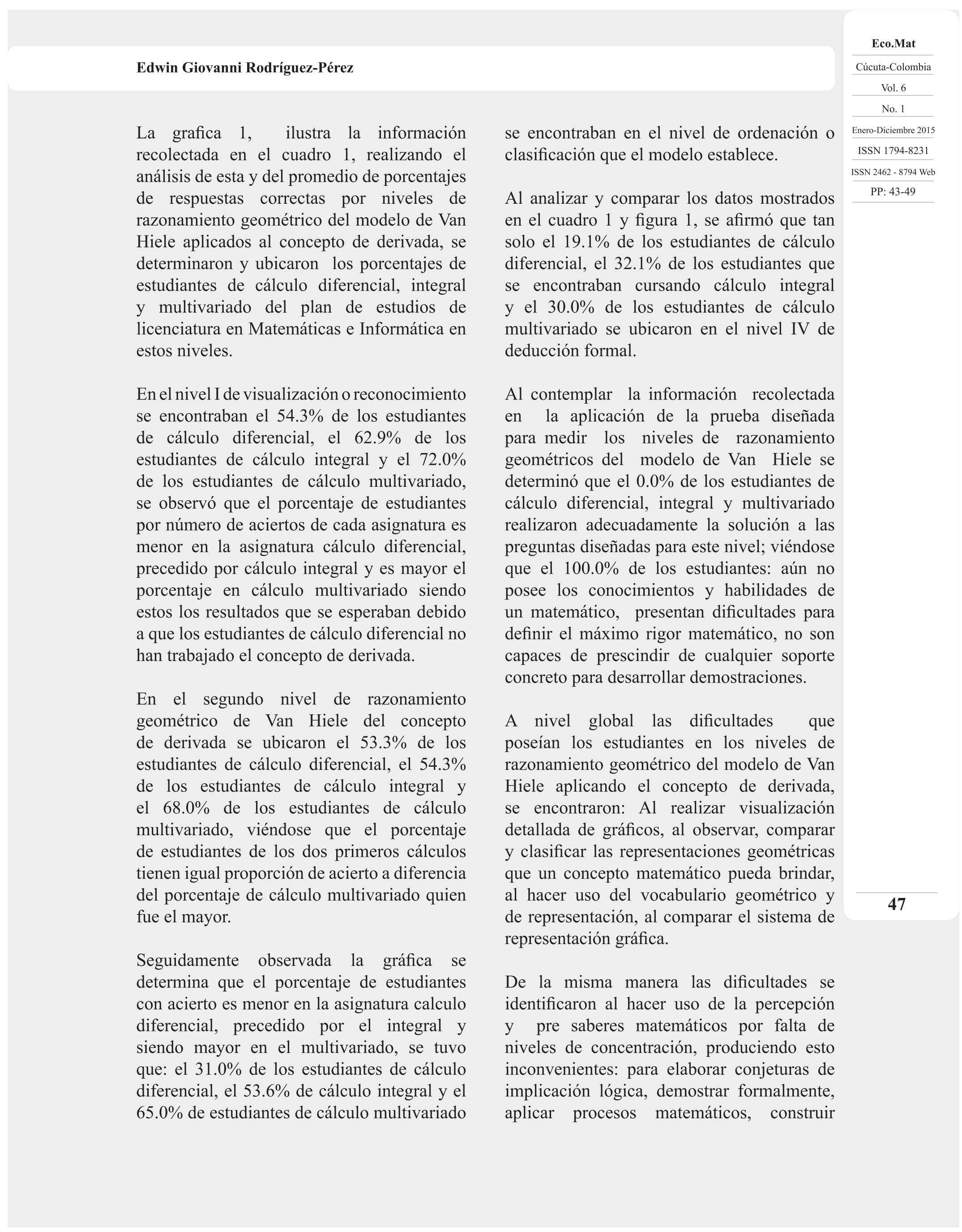


Eco.Mat

Cúcuta-Colombia

Vol. 6

No. 1

Enero-Diciembre 2015

ISSN 1794-8231

ISSN 2462 - $8794 \mathrm{We}$ PP: $43-49$

El concepto de derivada y el modelo de Van Hiele en estudiantes de licenciatura en matemáticas e informática de la Universidad Francisco de Paula Santander

conceptos propios, seguir pasos individuales de un método y trabajar las matemáticas en su forma abstracta.

\section{Conclusiones}

Se realizó la caracterización de los niveles de razonamiento geométrico del modelo de Van Hiele aplicados al concepto de derivada en los estudiantes de cálculo diferencial, integral y multivariado de Licenciatura en Matemáticas e Informática de la Universidad Francisco de Paula Santander; determinando, describiendo y comparando los diferentes niveles de razonamiento geométrico en el que se ubican los estudiantes.

En el nivel I de visualización se encontraban el $54.3 \%$ de los estudiantes de cálculo diferencial, el $62.9 \%$ de los estudiantes de cálculo integral y el $72.0 \%$ de los estudiantes de cálculo multivariado, y se identificó que los estudiantes: reconocían graficas en ilustraciones dadas; percibían los componentes de las figuras; realizaban observación de los prototipos visuales pero no reconocían que las representaciones dadas establecen la representación geométrica de la derivada; Percibían, describían e identificaban objetos de una figura y los reproducían; Manejaban y construían afirmaciones adecuadas con información intrínseca del concepto de derivada a través de información visual; hacían uso del vocabulario geométrico con alguna dificultad. Siendo estos los resultados que se esperaban debido a que los estudiantes de cálculo diferencial no han trabajado plenamente el concepto de derivada.

El $53.3 \%$ de los estudiantes de cálculo integral, el $54.3 \%$ de los estudiantes de cálculo integral y el $68.0 \%$ de los estudiantes de cálculo diferencial, se ubicaron en el segundo nivel de razonamiento geométrico de Van Hiele aplicado concepto de derivada, porque: hacían referencia al uso de la definición de recta pendiente, realizaban demostraciones informales; reconocían que las figuras poseen propiedades geométricas y que al hallarlas se debe hacer el uso de los procedimientos matemáticos; Establecieron conjeturas mediante la observación de las pendientes de las gráficas ilustradas; Realizaban inconscientemente generalizaciones, ejemplificaban para comprobar experimentalmente; Identificaron perfectamente el objetivo matemático que presentaba la ilustración.

En el nivel de clasificación se tuvo que: el $31.0 \%$ de los estudiantes de cálculo diferencial, el $53.6 \%$ de cálculo integral y el $65.0 \%$ de estudiantes de cálculo multivariado se encontraban en este nivel debido a que: entendían los pasos para calcular la pendiente de la recta tangente a una curva y el significado de la definición de pendiente de recta tangente a una función en un punto dado y ordenaron sus conceptos analizando el grafico mostrado; hacían uso adecuado de pasos dados en la información; De una manera experimental e intrínseca resolvieron la derivada de una función; Reconocieron e integraron que toda función tiene derivada en un punto, si el límite existe; y modificaron pasos de un procedimiento.

El análisis de las pruebas indica que tan solo el $19.1 \%$ de los estudiantes de cálculo diferencial, el $32.1 \%$ de los estudiantes de cálculo integral y el $30.0 \%$ de los estudiantes de cálculo multivariado se ubican en el nivel de deducción formal, debido a que: Dadas dos afirmaciones relacionadas pueden entender y realizar razonamientos lógicos formales; Realizan deducciones aceptando la existencia de la definición de la derivada; Establece conceptos alternativos al comparar las diferentes interpretaciones geométricas.

En el nivel de rigor, el $100.0 \%$ de los estudiantes a los que se le aplicó la prueba no poseían los conocimientos y habilidades de un matemático; Presentaron dificultades 
para definir el máximo rigor de abstracción; y no fueron capaces de prescindir de cualquier soporte concreto para desarrollar las demostraciones implicadas con las reglas básicas de derivación.

Se encontró en la aplicación de la prueba que los estudiantes de cálculo diferencial, integral y multivariado presentan constantes y diversas dificultades a la hora de realizar un razonamiento matemático trabajado en su concepción geométrica; las dificultades más notorias son: de percepción; de análisis e interpretación gráfica.

La investigación realizada mostró que es posible aplicar los niveles de razonamiento geométrico del modelo educativo de los esposos Van Hiele aun concepto propio de la Matemática, para evaluar el nivel en el que se encuentran los estudiantes siendo este el punto de partida para aplicar las fases de aprendizaje del mismo modelo para reestructurar $y$ mejorar un concepto previamente trabajado.

\section{Bibliografía}

Artigüe, M. (2009). La enseñanza de los principios del cálculo: problemas epistemológicos, cognitivos y didácticos. En Artigue, M.; Douady, R.; Moreno, L. Y Gómez, P. (editor). Ingeniería didáctica en educación matemática. Una Empresa Docente. Bogotá. Grupo Editorial Iberoamérica.

Corberan, R. y et al, (1994). Diseño y evaluación de una propuesta curricular de aprendizaje de la geometría en enseñanza secundaria basada en el nivel de razonamiento de Van Hiele. Primera edición. Editorial CIDE. Madrid (1994).pp. 11-29.

Esteban, P. (2003). Aspectos comparativos en la extensión del modelo de Van Hiele al campo de la aproximación local Universidad EAFIT, Medellín, Colombia, en: revista Suma44 $n^{\circ}$ 22, pp.45-52.
Fouz, F. Modelo de van hiele para la didáctica de la geometría. En: http://divulgamat.ehu. es/weborriak/testuakonline/04-05/pg-04-05fouz.pdf

Hernández, R. (2001). Metodología de la investigación. Segunda edición. Editorial McGraw-Hill. Interamericana, S.A.p 481

Llorens, J. (2003). Aspectos comparativos en la extensión del modelo de Van Hiele al campo de la aproximación local Universidad politécnica de Valencia, Valencia, España, en: revista Suma44 n ${ }^{\circ} 22$, pp.45-52.

Méndez, C. (2004). Metodología guía para elaborar diseños de investigación en ciencias económicas, contables y administrativas. Tercera edición. Editorial McGraw- Hill, Interamericana, S.A. Bogotá.246 p.

Ramírez, R. (2009). Universidades U.D.C.A y Libre/ponencia, Historia y epistemología de la función derivada, Tecné, Episteme y Didaxis: TED No. Extraordinario, $4^{\circ}$ Congreso Internacional sobre Formación de Profesores de Ciencias.

Stewart, J. Definición de función, limite, continuidad, derivada; mcmaster university international, Thomson, Editores. 57

Vrancken, S. y et al. (2008).Una propuesta para la introducción del concepto de derivada desde la variación. Análisis de resultados. Argentina en: http://www.soarem.org.ar/ Documentos/38\%20Vrancken.pdf. Artículos
Enero-Diciembre 2015 ISSN 1794-8231 ISSN 2462 - 8794 Web PP: $43-49$

Universidad nacional de litoral. 